\title{
Solving Linear Systems of Differential and Difference Equations with Respect to a Part of the Unknowns
}

\author{
S. A. Abramov* and M. Bronstein ${ }^{\dagger} * *$ \\ * Dorodnicyn Computing Center, Russian Academy of Sciences, \\ ul. Vavilova 40, Moscow, 119991 Russia \\ ** L'Institut National de Recherche en Informatique et en Automatique, \\ Sophia-Antipolis, France \\ e-mail:sabramov@ccas.ru \\ Received July 6, 2005
}

\begin{abstract}
The existence of solutions $y$ to a linear differential or difference system whose components $y_{i_{1}}, \ldots, y_{i_{m}}$ belong to a given class of functions and the problem of constructing such components are considered.
\end{abstract}

DOI: $10.1134 / \mathrm{S} 0965542506020059$

Keywords: computer algebra, linear systems of differential and difference equations, solution of a system with respect to a part of the unknowns

\section{INTRODUCTION}

Let $K$ be a differential field, for example, the field of rational functions with real or complex coefficients, and let $S$ be the system of differential equations

$$
y^{\prime}=A y,
$$

where $y=\left(y_{1}, \ldots, y_{n}\right)^{\mathrm{T}}$ and $A \in \operatorname{Mat}_{n}(K)$. Computer algebra provides many algorithms for constructing solutions to systems (1) belonging to $K$ and to its extensions. If $K$ is the field of rational functions, one can find solutions belonging to this field, solutions in the form of hyperexponential functions (i.e., functions with a rational logarithmic derivative), solutions in the form of algebraic or Liouville functions, and so on (see $[1,2])$. However, these algorithms find only the solutions $y$ for which all the components belong to the given class. In this paper, we consider the problem of constructing solutions with respect to a part of the unknowns. More precisely, assume that a set of components $\left\{y_{i_{1}}, \ldots, y_{i_{m}}\right\}$ of the vector $y$ is given and a class of functions $F$ (a linear space over the field $K$ closed with respect to the differentiation) is specified. We want to find the components of the solutions $y$ to system (1) for which all the specified components belong to $F$. For example, the problem can be as follows. For given system (1), where $K$ is the field of the rational functions and $n \geq 2$, find all the pairs of the rational functions that are the first and the second components of a solution $y=\left(y_{1}, \ldots, y_{n}\right)^{\mathrm{T}}$; the other components of the solution are of no interest.

In computer algebra, solutions in the form of a power series are also of interest (an algorithm for constructing such a solution must be able to produce any number of the series terms). An example is provided by regular formal solutions to a differential system (see [3-5]), where, e.g., $K$ is the field of rational functions with complex coefficients. In a neighborhood of a fixed point $x_{0}$, these solutions have the form

$$
\left(x-x_{0}\right)^{\lambda} \sum_{v=0}^{k} \varphi_{v}\left(x-x_{0}\right) \log ^{v}\left(x-x_{0}\right),
$$

where $\varphi_{v}\left(x-x_{0}\right)$ are the Laurent series and $\lambda \in \mathbb{C}$. In a neighborhood of an irregular singular point, the system does not always have a solution with all regular components; in this context, it makes sense to consider the problem discussed in this paper.

We also can consider the classes of functions that are not represented in a "closed" form even in the broadest sense of this word; for example, we can consider meromorphic functions.

\footnotetext{
$\dagger$ Deceased.
} 
In Section 3, we propose an algorithm that yields one of the following two possible results:

(1) The conclusion that if the specified components of a solution belong to the given class $F$, then all the other components also belong to this class.

(2) A new system of a smaller order in which the specified components of a solution to the initial system and some of their derivatives are the unknowns; if the specified components of the initial system belong to the given class $F$, then all the other components of the new system also belong to this class.

The problem of the partial construction of solutions is in keeping with the problem of observability and stability of solutions with respect to a part of the variables (see [6-8]). In Section 4, we discuss this issue and compare our approach with the approach (which is used in mechanics) based on $\mu$-transformations proposed in [7]. It should be noted that the new basis chosen for the transformation of the system includes vectors belonging to the set that was considered in [8] in connection with the problems of stability and observability. However, the partial construction of solutions was not considered in the works cited above.

In Section 2, we introduce the concept of the $m$-rank of a system and an admissible partition of the $m$ rank; these concepts are used to validate our approach. In Section 4, we separately consider the case $m=1$, i.e., the case when we are interested only in a single component of the solution. We show that the linear homogeneous equation of minimal order for $y_{1}$ that is a consequence of system (1) has no extraneous solutions (to obtain this equation, we construct linear forms that represent $y_{1}, y_{1}^{\prime}, y_{1}^{\prime \prime}, \ldots$ in terms of $y_{1}, \ldots, y_{n}$ until they become linearly dependent). The statement in [9, Section 1.2] to the effect that an equation of order not exceeding $n$ with respect to $y_{j}(1 \leq j \leq n)$ "usually has many solutions which are not the $j$ th component of a solution of the initial system" is true only when the order of this equation is not minimal.

In Section 6, the concept of an admissible partition of the $m$-rank is used to formulate and prove a local existence theorem for nonlinear autonomous systems. In Section 7, difference systems are discussed. In Section 8 , we briefly discuss the description and implementation of the algorithm in terms of noncommutative Ore polynomials, which makes it possible to develop a universal computer program adjustable to the differential, difference, and some other cases.

In the further presentation, we assume, for simplicity, that $\left(i_{1}, \ldots, i_{m}\right)=(1,2, \ldots, m)$. Thus, we are interested in the components $\left(y_{1}, \ldots, y_{m}\right)(m \leq n)$ of the solution to the system $S$. We also assume that, in addition to the class $F$, there exists a class $G(F \subseteq G)$ which is also a linear space over $K$ such that any system $y^{\prime}=$ $A y+b$ with $A \in \operatorname{Mat}_{n}(K)$ and $b \in F^{n}$ has at least one solution in $G^{n}$, i.e., a solution with all the components belonging to $G$. (We do not specify this class and do not discuss its structure; rather, we only assume that such a class exists.) This assumption is quite natural; it relieves us of the necessity to consider the issues of differential algebra related to the Picard-Vessio extensions (see [2]) and so on. When we mention solutions to the system without specifying the class to which they belong, solutions with components in $G$ are assumed. The elements of the spaces $F$ and $G$ are called functions, although a more formal differential-algebraic interpretation of these spaces is possible.

\section{ADMISSIBLE PARTITIONS OF THE $m$-RANK OF A SYSTEM}

Let $e_{1}, \ldots, e_{n}$ be the standard basis in the space of the linear forms of $y_{1}, \ldots, y_{n}$ over $K$ :

$$
e_{1}=y_{1}, \ldots, e_{n}=y_{n} .
$$

Every linear form $f$ can be differentiated along the solutions to the system $S$, which yields another linear form of $y_{1}, \ldots, y_{n}$. Identifying any linear form with the column vector of its coefficients, we obtain for the derivatives along the solutions to the system $S$ the representation

$$
f^{(0)}=f, \quad f^{(i)}=A^{\mathrm{T}} f^{(i-1)}+\frac{d}{d x} f^{(i-1)}, \quad i=1,2, \ldots,
$$

where $\frac{d}{d x} f^{(i-1)}$ is the result of the componentwise differentiation of the column vector $f^{(i-1)}$.

The dimension $l$ of the linear $\operatorname{space}^{\operatorname{Span}_{m}}(S)$ of the linear forms generated by $e_{1}, \ldots, e_{m}$ and by all the linear forms $e_{i}^{(j)}(i=1,2, \ldots, m, j=1,2, \ldots)$ is called the $m$-rank of the system $S$. If the nonnegative integers 
$l_{1}, \ldots, l_{m}$ are such that the linear forms

$$
\begin{gathered}
e_{1}, e_{1}^{(1)}, \ldots, e_{1}^{\left(l_{1}-1\right)}, \\
\ldots . \ldots . \ldots . \ldots . . . \\
e_{m}, e_{m}^{(1)}, \ldots, e_{m}^{\left(l_{m}-1\right)}
\end{gathered}
$$

form a basis

$$
f_{1}, \ldots, f_{l}
$$

in the $\operatorname{space}^{\operatorname{Span}_{m}}(S)\left(l=l_{1}+\ldots+l_{m}\right)$, then the ordered set $\left(l_{1}, \ldots, l_{m}\right)$ is an admissible partition of the $m$ rank of the system $S$.

Example 1. Consider the system $y^{\prime}=A y$ consisting of three equations with constant coefficients in which

$$
A=\left(\begin{array}{lll}
0 & 0 & 1 \\
0 & 1 & 0 \\
1 & 0 & 0
\end{array}\right) .
$$

The 2-rank $l$ of this system is equal to three, and the only admissible partition of $l$ is $(2,1)$. For example, the partition $(1,2)$ is inadmissible because $e_{2}^{\prime}=e_{2}$. On the other hand, the 3-rank of this system has three admissible partitions: $(2,1,0),(0,1,2)$, and $(1,1,1)$.

The derivative of any linear form in (3) along the solutions to the system $S$ (i.e., the derivative of any linear form in $f_{1}, \ldots, f_{l}$ ) is a linear combination (over $K$ ) of the linear forms $f_{1}, \ldots, f_{l}$. Introduce new variables $z_{1}, \ldots, z_{l}$ that are represented in terms of $y_{1}, \ldots, y_{n}$ by the linear forms $f_{1}, \ldots, f_{l}$. Let the matrix $B \in \operatorname{Mat}_{l}(K)$ be such that the column vector of the coefficients of the derivative of the linear form $f_{i}$ along the solutions to the system $S$ is equal to the product of $B$ and the column vector of the coefficients $f_{i}(i=1,2, \ldots, l)$. Set up the system of equations

$$
z^{\prime}=B z
$$

where $z=\left(z_{1}, \ldots, z_{l}\right)^{\mathrm{T}}$. The basis $f_{1}, \ldots, f_{l}$ can be extended to the basis

$$
f_{1}, \ldots, f_{l}, f_{l+1}, \ldots, f_{n}
$$

of the space of all linear forms of $y_{1}, \ldots, y_{n}$. The use of the linear forms from this basis for introducing the new variables $Z=\left(z_{1}, \ldots, z_{l}, z_{l+1}, \ldots, z_{n}\right)$ corresponds to the change of variables

$$
Z=H y
$$

with a nonsingular matrix $H \in \operatorname{Mat}_{n}(K)$. The initial system $S$ can be reduced to the form

$$
Z^{\prime}=\left(\begin{array}{cc}
B & 0 \\
U & V
\end{array}\right) Z .
$$

Proposition 1. (i) The projections of the set of solutions to the initial system $S$ and to the system $z^{\prime}=B z$ on $y_{1}, \ldots, y_{m}$ are identical.

(ii) If a solution to the system $z^{\prime}=B z$ is such that its components $y_{1}, \ldots, y_{m}$ belong to the given class $F$, then all the components of this solution belong to this class.

(iii) If the m-rank of the initial system is $n$ and this system has a solution with the components $y_{1}, \ldots, y_{m}$ belonging to the given class $F$, then all the components of this solution belong to this class.

Proof. (i) Set $z=\left(z_{1}, \ldots, z_{l}\right)^{\mathrm{T}}$ and $\tilde{z}=\left(z_{l+1}, \ldots, z_{n}\right)^{\mathrm{T}}$. Using the solution $z$ to system (4) and system (5), we obtain the following nonhomogeneous system for $\tilde{z}$ :

$$
\tilde{z}^{\prime}=V \tilde{z}+U z
$$

According to the assumption made at the end of the Introduction, this system has a solution in the class $G$. On the other hand, $m$ unknowns from $z_{1}, \ldots, z_{l}$ are equal to $y_{1}, \ldots, y_{m}$ (because the transformation $H$ does not 
change the values of these unknowns):

$$
\begin{gathered}
z_{1}=y_{1}, \quad z_{2}=y_{1}^{\prime}, \quad \ldots, \quad z_{l_{1}}=y_{1}^{\left(l_{1}-1\right)}, \\
\ldots \ldots \ldots \ldots \ldots \ldots \ldots \ldots \ldots \ldots \ldots \ldots \ldots \ldots \ldots \ldots \ldots \ldots \ldots \ldots \ldots \ldots \ldots \\
z_{k}=y_{m}, \quad z_{k+1}=y_{m}^{\prime}, \quad \ldots, \quad z_{l}=y_{m}^{\left(l_{m}-1\right)} .
\end{gathered}
$$

(ii) This assertion is obvious because the class $F$ is closed with respect to the differentiation.

(iii) In this case, system (5) has the form $Z=B Z$ and $y=H^{-1} Z$. Then, use (ii).

Setting $F=\{0\}$, we obtain two corollaries to Proposition 1 .

Corollary 1 . The initial system has a nonzero solution with the zero components $y_{1}, \ldots, y_{m}$ if and only if its $m$-rank is less than $n$.

Corollary 2. All the components of a nonzero solution to the irreducible (over $K$ ) system (i.e., to a system that cannot be reduced to form (5) by a linear (over $K$ ) nonsingular change of unknowns) are distinct from zero.

For example, if we are interested in rational or meromorphic solutions to the system $z^{\prime}=B z$, then the fact that some of the unknowns are derivatives of others can be used to reduce the a priori bounds of the orders of the poles of the solutions (such bounds can be obtained using the methods studied in [10,11]). For exam-

ple, if we know that $z_{1}^{\prime}=z_{2}, z_{2}^{\prime}=z_{3}, \ldots, z_{l-1}^{\prime}=z_{l}$ and that $\gamma>0$ is an upper bound on the orders of the poles of the unknown functions $z_{1}, \ldots, z_{l}$ at the point $x=x_{0}$, then we can use the improved bounds $\gamma-l, \gamma-l+1, \ldots$, $\gamma-1$ on the orders of the poles of the unknown functions $z_{1}, \ldots, z_{l-1}$, respectively. If it turns out that $\gamma-l \leq 0$, then none of the functions $z_{1}, \ldots, z_{l}$ has a pole at $x_{0}$.

\section{ALGORITHM}

Computation of the $m$-rank of the initial system and the construction of the system $z^{\prime}=B z$ of form (4) play a key role in the solution of the problem.

The algorithm is as follows. If the $m$-rank of the initial system is $n$, then the algorithm concludes that, since the specified components of the solution to the system belong to the given class, then all the components also belong to this class. If the $m$-rank of the initial system is less than $n$, then set up the system $z^{\prime}=$ $B z$ as described in Section 2, find its solutions with all the components belonging to the given class, and produce the corresponding subset of the components of these solutions (removing the derivative components).

Here, it is pertinent to note that, as was mentioned in the Introduction, computer algebra provides many algorithms for constructing solutions to systems (1) with all the components belonging to a certain class of functions. These algorithms can be divided into two types. The first type includes the algorithms that reduce the initial system to a scalar equation of order $n$. Such a reduction is called uncoupling. Usually, it is performed by constructing a cyclic vector, which will be briefly discussed in the next section. The second type comprises all the other methods, which are called direct. These algorithms are faster; however, they are few in number. For the case when $K$ is the field of rational functions, three such algorithms are based on the search for rational solutions $[11,10]$, for formal regular solutions $[4,5]$, and for irregular solutions with the use of the Puiseux series [12]. However, the algorithms for finding Liouville solutions (or, which is the same, solutions by quadratures) [2] can be used only in the scalar case; in the multidimensional case, uncoupling is used.

The algorithm described in this section is designed for use at the preliminary stage, which precedes the solution of the system $z^{\prime}=B z$ (this system can be called an $m$-system) with all the components belonging to the given class.

We complete the discussion of the algorithm by a remark concerning the case when the $m$-rank of the initial system is $n$. In this case, the matrix $B$ can also be constructed, and its size is $n \times n$. However, the algorithm recommends skipping the construction of $B$ and returning to the initial system $y^{\prime}=A y$ (the operations used to transform $A$ into $B$ can make the matrix entries more bulky). However, if we do not assume that $F$ is a linear space over $K$ but is only closed with respect to differentiation (this problem is slightly different from the one considered in this paper), the transition to the system $z^{\prime}=B z$ is appropriate even when the $m$ rank is equal to $n$. In this case, part (iii) of Proposition 1 does not apply because $H^{-1} z$ can contain the components not belonging to $F$. For example, this is the case when $K=\mathbb{Q}(x)$ and we are interested in the polynomial components $y_{1}, \ldots, y_{m}$ or in the same components in the form of entire functions. For example, for 
the system

$$
y^{\prime}=\left(\begin{array}{cc}
-1 / x & 2 x \\
0 & -1 / x
\end{array}\right) y,
$$

we have $n=2, m=1$, and its 1 -rank is 2 . The solutions $(x, 1 / x)^{\mathrm{T}}$ and $(1 / x, 0)^{\mathrm{T}}$ to this system are linearly independent, which implies that this system has no solutions in which both components are polynomial. The consideration of the initial system instead of $z^{\prime}=B z$ does not make it possible to find the polynomial component $y_{1}=x$. In this case,

$$
B=\left(\begin{array}{cc}
0 & 1 \\
1 / x^{2} & -1 / x
\end{array}\right)
$$

and the solutions of the corresponding system with both polynomial components are represented as the product of an arbitrary constant and $(x, 1)^{\mathrm{T}}$.

Usually, the search for polynomial solutions is performed in two steps. At the first step, an a priori upper bound for the degrees of the polynomials that are the components of any such solution is found. At the second stage, these components are found using, for example, the method of undetermined coefficients. Here, we can make a simplification similar to that described at the end of Section 2 . If it is known that the degree of any of the polynomials $z_{1}, \ldots, z_{l}$ is less than $\gamma \geq 0$ and $z_{1}^{\prime}=z_{2}, \ldots, z_{l-1}^{\prime}=z_{l}$, then $\operatorname{deg} z_{2} \leq \gamma-1, \operatorname{deg} z_{3} \leq \gamma$ $-2, \ldots, \operatorname{deg} z_{l} \leq \gamma-l+1$. If, for a certain $i$ in the range $1 \leq i \leq l-1, \gamma-1<0$, then $z_{i+1}=\ldots=z_{l}=0$.

\section{THE CASE WHERE $m=1$}

If $m=1$, then the matrix $B$ is

$$
\left(\begin{array}{cccc}
0 & 1 & & \\
& & \ddots & \\
& & & 1 \\
g_{0} & g_{1} & \ldots & g_{l-1}
\end{array}\right),
$$

where $l$ is equal to the 1 -rank of the initial system. In this case, the set of solutions to the system $z^{\prime}=B z$ obviously coincides with the set of the vectors $\left(u, u^{\prime}, \ldots, u^{(l-1)}\right)^{\mathrm{T}}$, where $u$ ranges over all the solutions to the equation $L u=0$ with

$$
L=\frac{d^{l}}{d x^{l}}-\sum_{i=0}^{l-1} g_{i} \frac{d^{i}}{d x^{i}} .
$$

Applying Proposition 1 to the case $m=1$, we see that any $y_{1}=u$, where $u$ is a solution to the equation $L u=0$, can be extended to become a solution of the system $y^{\prime}=A y$. Thus, we have the following result.

Proposition 2. All the solutions to the equation $L u=0$ and only these solutions are the $y_{1}$ components of all the solutions $y$ of the system $y^{\prime}=A y$

Note that if the 1-rank of system (1) is $n$, then $y_{1}$ is the so-called cyclic vector (see [13]) for this system. The validity of Proposition 2 for this case has always been beyond question; the misunderstanding mentioned in the Introduction in connection with the quotation from [9] concerned the case of smaller values of the $m$-rank. A cyclic vector of system (1) is a linear form $v=v_{1} y_{1}+\ldots+v_{n} y_{n}$ with the coefficients from $K$ such that $v$, in combination with the derivatives $v^{\prime}, \ldots, v^{(n-1)}$ along the solutions of the system, form a basis

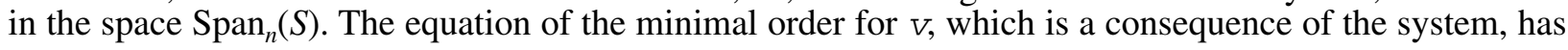
the order $n$ in this case. It is proved that, under broad assumptions about the field $K$, a cyclic vector exists, and there are algorithms for its construction. One of such algorithms is the one based on a random choice of the coefficients $v_{1}, \ldots, v_{n}$ with the subsequent verification of the linear independence of the set $v, v^{(1)}$, $\ldots, v^{(n-1)}$. If necessary, this procedure is repeated until a cyclic vector is found.

In the following examples, in the consideration of a problem with respect to a part of the unknowns, we usually assume that the field of the system's coefficients $K$ and the class $F$ consist of the rational functions. This is done only for the sake of simplicity: our approach does not rely on this assumption. 
Example 2. Consider the system $y^{\prime}=A y$, where

$$
A=\left(\begin{array}{ccc}
3 x & -1-2 x & 2 x \\
\left(1-x-x^{2}\right) / x^{2} & -1 / x^{2} & \left(1-x-x^{2}\right) / x^{2} \\
\left(1-x-3 x^{3}\right) / x^{2} & \left(x^{2}-1+2 x^{3}\right) / x^{2} & \left(1-x-2 x^{3}\right) / x^{2}
\end{array}\right),
$$

$y=\left(y_{1}, y_{2}, y_{3}\right)^{\mathrm{T}}$, and $K=\mathbb{Q}(x)$. Assume that we are interested in all rational $y_{1}$ and $y_{2}$. The 2-rank of this system is 3 . Therefore, if the components $y_{1}$ and $y_{2}$ of a solution to this system belong to an appropriate class (in particular, to the class of rational functions), then all the components of this solution also belong to this class. The direct algorithm described in [10] applied to finding the rational solutions shows that this system has no nonzero rational solutions; therefore, it has no nonzero solutions for which both components $y_{1}$ and $y_{2}$ are rational functions. If we are interested in all possible rational $y_{2}$, then the corresponding 1-rank is 2 and the matrix $B$ in the new system $z^{\prime}=B z$ is

$$
B=\left(\begin{array}{cc}
0 & 1 \\
(x+3) /\left(x^{3}+x^{2}-x\right) & (x+3)(1-x) /\left(x^{3}+x^{2}-x\right)
\end{array}\right),
$$

$z=\left(z_{1}, z_{2}\right)^{\mathrm{T}}, z_{1}=y_{2}$, and $z_{2}=y_{2}^{\prime}$. The rational solutions to the system $z^{\prime}=B z$ can be found using the direct algorithm described in [10]. It is also seen that, according to Proposition 2, this system is equivalent to the scalar equation $L y_{2}=0$, where

$$
L=\left(x-x^{2}-x^{3}\right) \frac{d^{2}}{d x^{2}}+(x+3)(1-x) \frac{d}{d x}+x+3 .
$$

The projection of the space of solutions to the system $y^{\prime}=A y$ on $y_{2}$ coincides with the space of solutions to Eq. $L y_{2}=0$, which, as can be shown, has the basis $x-1, x^{-1}+x^{-2}$. Therefore, Eq. $L y_{2}=0$ has no other solutions except for the rational ones; $y_{2}$ is a rational function for all the solutions of $y^{\prime}=A y$; and, for any constants $c_{1}$ and $c_{2}$, there is a solution $y$ such that $y_{2}=c_{1}(x-1)+c_{2}\left(x^{-1}+x^{-2}\right)$. The space of solutions to this system for which $y_{2}=0$ is one-dimension because system (6) consists of a single first-order equation in this case. It can be shown that, in complete agreement with Corollary 1 to Proposition 1 , the one-dimensional space of the solutions for which $y_{2}=0$ is generated by the solution

$$
y=e^{x^{2} / 2}\left(\begin{array}{c}
1 \\
0 \\
-1
\end{array}\right)
$$

If a direct algorithm is used to find the rational solutions to the system $z^{\prime}=B z$ with matrix (8), then a preliminary estimation of the order of the poles can yield, for example, the upper bound of 3 for $x=0$ and 1 for the roots of the equation $x^{2}+x-1=0$. The change of variables

$$
z_{1}=\frac{p_{1}(x)}{x^{3}\left(x^{2}-x+1\right)}, \quad z_{2}=\frac{p_{2}(x)}{x^{3}\left(x^{2}-x+1\right)}
$$

in $z^{\prime}=B z$ yields a very cumbersome system of equations for finding the polynomials $p_{1}$ and $p_{2}$. The solution to this system yields sixth-degree polynomials such that rational functions (9) turn out to be reducible by $x\left(x^{2}-x-1\right)$ and $x^{2}-x-1$, respectively. Using the reasoning presented at the end of Section 2 , we obtain that the solutions to the system $z^{\prime}=B z$ cannot have poles at the roots of the equation $x^{2}+x-1=0$ and a bound on the order of the pole $z_{1}$ at 0 is 2 . Therefore, one can use the change of variables $z_{1}=p_{1} / x^{2}, z_{2}=p_{2} / x^{3}$ instead of (9), which results in a simpler system that has the cubic polynomials $p_{1}(x)$ and $p_{2}(x)$ as its solutions. 


\section{STRUCTURE OF THE MATRIX $B$ AND PARTIAL STABILITY OF THE ZERO SOLUTION IN THE CASE OF A SYSTEM WITH CONSTANT COEFFICIENTS}

We have already seen that, for $m=1$, the matrix of the system $z^{\prime}=B z$ is a companion matrix of form (7). Consider the case $m>1$, in which $B$ is an $l$-by- $l$ matrix of the form

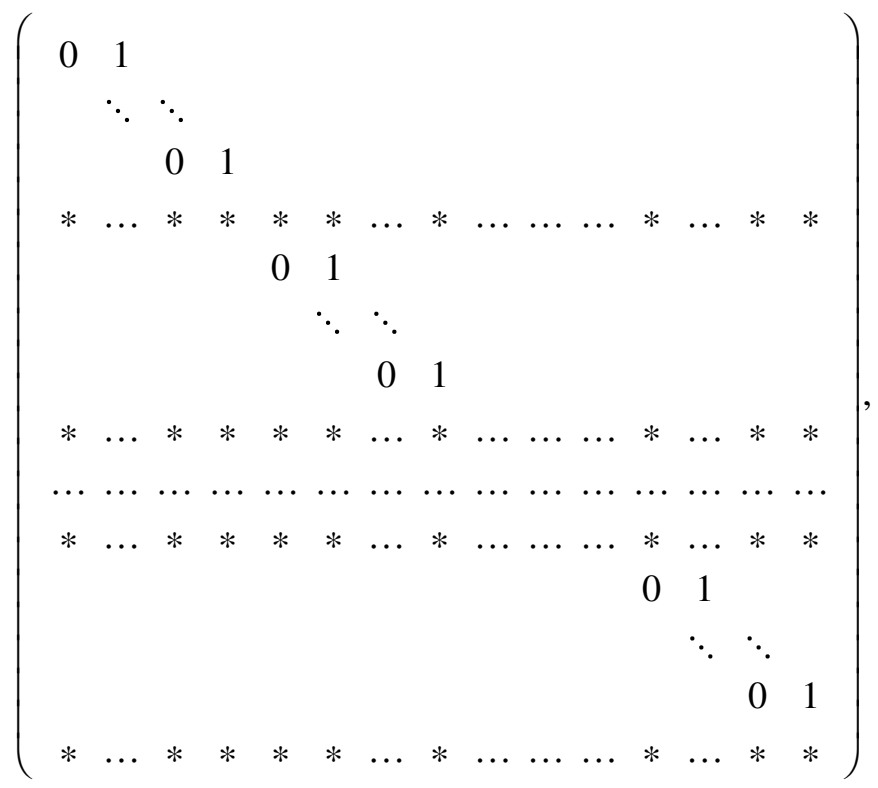

where the asterisks denote elements of the field $K$. If $A$ in the initial system is a constant $n$-by- $n$ matrix, then $B$ is also constant.

The direct application of the Routh-Hurwitz criterion does not help to analyze the stability of the zero solution to the system $y^{\prime}=A y$ with a constant real matrix $A$ with respect to $y_{1}, \ldots, y_{m}$ (this is a well-known problem in classical mechanics, see [6]).

Example 3. Consider the system $y^{\prime}=A y$ with

$$
A=\left(\begin{array}{ccc}
-1 & 1 & -2 \\
4 & 1 & 0 \\
5 / 2 & 1 & -1
\end{array}\right)
$$

Since the characteristic polynomial has a root 1 , the solution $y_{1}=y_{2}=y_{3}=0$ is Lyapunov unstable. We want to analyze the stability of this system with respect to $y_{1}$. Applying the $\mu$-transformation method proposed in [7] (see also [6]), we define $\mu_{1}=y_{2}-2 y_{3}$ in the first equation

$$
y_{1}^{\prime}=-y_{1}+y_{2}-2 y_{3}
$$

of the initial system. Then, we obtain

$$
\mu_{1}^{\prime}=-y_{1}-y_{2}+2 y_{3} .
$$

Since the 1-rank of the initial system is 2 , the unknowns $y_{2}$ and $y_{3}$ can be eliminated. We have

$$
\begin{aligned}
& y_{1}^{\prime}=-y_{1}+\mu_{1}, \\
& \mu_{1}^{\prime}=-y_{1}-\mu_{1} .
\end{aligned}
$$

In the general case, it can be necessary to use $\mu_{1}, \mu_{2}, \ldots$ instead of $\mu$. The characteristic polynomial of the last system is $-\lambda^{2}-2 \lambda-2$; its roots are $-1+i$ and $-1-i$. The real part of each of them is negative; therefore, the solution $y_{1}=y_{2}=y_{3}=0$ to the initial system is asymptotically stable with respect to $y_{1}$. 
The consideration of the system $z^{\prime}=B z$ described in Section 2 can be used instead the $\mu$-transformation. In the example under consideration, we have

$$
B=\left(\begin{array}{cc}
0 & 1 \\
-2 & -2
\end{array}\right),
$$

$z_{1}=y_{1}, z_{2}=y_{1}^{\prime}$, and the roots of the characteristic polynomial are $-1+i$ and $-1-i$ as in the case of the $\mu$-transformation.

In the general case, form (10) of the matrix $B$ is convenient when the characteristic polynomial must be found. A permutation of the rows of the matrix $B-\lambda I_{l}$ reduces the problem to the computation of the determinant of the matrix

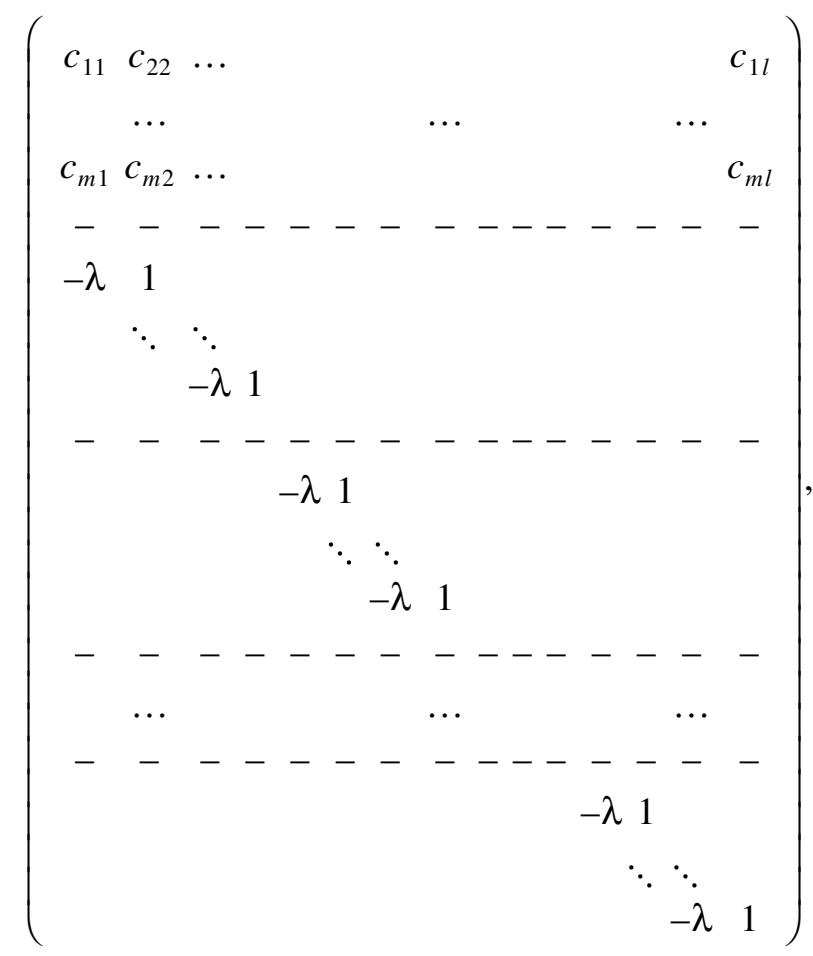

where the upper horizontal part consists of $m$ rows and the subsequent parts consist of $l_{1}-1, \ldots, l_{m}-1$ rows, where $l_{1}+\ldots+l_{m}=l$. Eliminating the entries in the upper part of this matrix using the unit entries of the subsequent rows (first, $c_{1 n}, \ldots, c_{m l}$ are eliminated using the last row; then the next to last row is used; and so on) and expanding the determinant in terms of the columns, of which each contains only one (unit) entry, we reduce the problem to calculating the determinant of the $m$-by- $m$ matrix $\left(p_{i j}(\lambda)\right)$ in which

$$
p_{i j}=c_{i, k_{j-1}+1}+c_{i, k_{j-1}+2} \lambda+\ldots+c_{i, k_{j}} \lambda^{l_{j}-1},
$$

where $k_{0}=0$ and $k_{j}=l_{1}+\ldots+l_{j}, j=1,2, \ldots, m$. This determinant, which is equal to the determinant of matrix (11) up to a sign, can be calculated by the standard methods. Thus, there is no need for writing out and storing the entire matrix (10); it is sufficient to find only the $m$ rows of this matrix depicted by the asterisks and construct the $m$-by- $m$ matrix $\left(p_{i j}(\lambda)\right)$.

Proposition 3. Suppose that the system $S$ of form (1) with constant coefficients has the $m$-rank $l(l \geq m \geq$ 1), the space $\operatorname{Span}_{m}(S)$ has basis (3), and the vector of the coefficients of the expansion $e_{s}^{\left(l_{s}\right)}$ in terms of this basis is $\left(d_{s 1}, \ldots, d_{s l}\right)$. Then, the characteristic polynomial of the matrix $B$ of system (4) produced by the proposed algorithm coincides, up to a sign, with the determinant of the m-by-m matrix $\left(p_{i j}(\lambda)\right)$ in which

$$
p_{i j}=d_{i, k_{j-1}+1}+d_{i, k_{j-1}+2} \lambda+\ldots+d_{i, k_{j}} \lambda^{l_{j}-1}-\lambda^{l_{j}},
$$

where $k_{0}=0$ and $k_{j}=l_{1}+\ldots+l_{j}, j=1,2, \ldots, m$. 
Proof. The proof immediately follows from the reasoning above concerning the calculation of the determinant of matrix (11): we set $c_{s t}=d_{s t}-\lambda$ if $t=k_{j}$ for a certain $j \leq m$ and set $c_{s t}=d_{s t}$ otherwise.

Proposition 3 is a generalization of the fact that if matrix (7) is constant, then its characteristic polynomial is equal to $g_{0}+g_{1} \lambda+\ldots+g_{l-1} \lambda^{l-1}-\lambda^{l}$ up to a sign.

For the case of constant coefficients, G.V. Plotnikov implemented the proposed algorithm for constructing the matrix $B$ and for finding the characteristic polynomial in Maple. Experiments showed that Plotnikov's program computes the characteristic polynomial of a matrix $B$ of order 20 with $m=3$ at least two and a half times faster than the standard Maple procedures.

The fact that, in the case of systems with constant coefficients, the stability of the zero solution to the initial system with respect to $y_{1}, \ldots, y_{m}$ is equivalent to the stability of the zero solution to the system $z^{\prime}=B z$ with respect to all of its variables (this is also true for the $\mu$-transformation method) can be derived from the fact that the solutions to the systems with constant coefficients have a special (well-known) form. However, in the case of variable coefficients, this is not always true.

Example 4. The equation

$$
(1+x) x y^{\prime \prime}-2 y^{\prime}+\left(9 x^{6}+9 x^{5}-2\right) y=0
$$

has the solutions $\left(\sin x^{3}\right) /(1+x)$ and $\left(\cos x^{3}\right) /(1+x)$. We can set up a linear system of two first-order equations for $y_{1}=y, y_{2}=y^{\prime}$. The zero solution to this system is obviously stable with respect to $y_{1}$ as $x \longrightarrow+\infty$; however, it is unstable with respect to $y_{1}, y_{2}$ : for example, the function $\left[\left(\sin x^{3}\right) /(1+x)\right]$ ' is unbounded as $x \longrightarrow+\infty$. If we apply to this system the transformation described in Section 2, the system does not change.

\section{6. $m$-RANK OF A NONLINEAR AUTONOMOUS SYSTEM}

The quantity that we call the $m$-rank in this paper is used in mechanics and control theory (although in a different manner) for the investigation of the observability of the system $y^{\prime}=A y$ in terms of the variables $y_{1}, \ldots, y_{m}$; here, $A \in \operatorname{Mat}_{n}(K)$. In a certain sense, the system is observable if and only if its $m$-rank is equal to $n$.

The concept of an admissible partition of the $m$-rank proposed in this paper can find other applications. We consider its application in the theory of autonomous systems of the form

$$
\begin{gathered}
y_{1}^{\prime}=f_{1}\left(y_{1}, \ldots, y_{n}\right), \\
\ldots \ldots \ldots . . . \ldots . . . \\
y_{n}^{\prime}=f_{n}\left(y_{1}, \ldots, y_{n}\right)
\end{gathered}
$$

such that $y_{1}(x)=\ldots=y_{n}(x)=0$ is a solution to this system; i.e.,

$$
f_{i}(0, \ldots, 0)=0, \quad i=1,2, \ldots, n .
$$

Assume that the functions $f_{1}, \ldots, f_{n}$ are sufficiently smooth. Then, $\left|y_{i}\right|<r(i=1,2, \ldots, n)$, where $r$ is a positive real number. For $0<m \leq n$, define the $m$-rank of system (12) as the $m$-rank (over $\mathbb{R}$ or $\mathbb{C}$ ) of its linearization $y^{\prime}=C y(C$ is a constant $n$-by- $n$ matrix $)$.

Proposition 4. Suppose that the m-rank of system (12) is $l$ and $\left(l_{1}, \ldots, l_{m}\right)$ is an admissible partition of $l$. Then, there exists a $\rho, 0<\rho<r$, such that, for any $a_{1}, \ldots, a_{m}$ satisfying the inequalities $\left|a_{i}\right|<\rho(i=1,2, \ldots$, $m)$, system (12) has a solution $\left(y_{1}, \ldots, y_{n}\right)^{\mathrm{T}}$ for which

$$
y_{i}(0)=a_{i}, \quad i=1,2, \ldots, m,
$$

and

$$
y_{i}^{(j)}(0)=0, \quad i=1,2, \ldots, m, \quad j=1,2, \ldots, l_{i}-1 .
$$

Proof. We consider only the case $m=1$. For the general case, the proof is similar. Denote by $Q\left(y_{1}, \ldots\right.$, $y_{n}$ ) the set of functions of the variables $y_{1}, \ldots, y_{n}$ such that each of these functions is defined in a neighborhood of the point $(0, \ldots, 0)$ and has the form

$$
\sum_{i=1}^{n} \sum_{j=1}^{n} \alpha_{i j}\left(y_{1}, \ldots, y_{n}\right) y_{i} y_{j}
$$


with sufficiently smooth $\alpha_{i j}$.

Assume that the first equations in the system have the form

$$
y_{k}^{\prime}=y_{k+1}+\varphi_{k}\left(y_{1}, \ldots, y_{n}\right),
$$

where $\varphi_{k} \in Q\left(y_{1}, \ldots, y_{n}\right), k=1,2, \ldots, l-1$. Using the induction on $k$, it can be easily proved that

$$
y_{1}^{(k)}=y_{k+1}+\psi_{k}\left(y_{1}, \ldots, y_{n}\right),
$$

$\psi_{k} \in Q\left(y_{1}, \ldots, y_{n}\right), k=1,2, \ldots, l-1$ (due to (13), the differentiation of any function in $Q\left(y_{1}, \ldots, y_{n}\right)$ with respect to $x$ yields, due to system (12), a function in $Q\left(y_{1}, \ldots, y_{n}\right)$ ). Substitute $y_{1}^{(k)}=0$ in (15) to obtain

$$
y_{k+1}+\psi_{k}\left(y_{1}, \ldots, y_{n}\right)=0,
$$

$k=1,2, \ldots, l-1$. Set $\Psi_{k}\left(y_{1}, \ldots, y_{n}\right)=y_{k}+\psi_{k-1}\left(y_{1}, \ldots, y_{n}\right), k=2,3, \ldots, l$. Obviously,

$$
\Psi_{k}(0, \ldots, 0)=0 \text { and }\left.\frac{\partial \Psi_{k}}{\partial y_{i}}\right|_{y_{1}=\ldots=y_{n}=0}=\delta_{k i},
$$

for $k=2,3, \ldots, l$ and $i=1,2, \ldots, n$. By the implicit function theorem, the equations $\Psi_{k}\left(y_{1}, \ldots, y_{n}\right)=0, k=2$, $3, \ldots, l$, make it possible to represent $y_{2}, \ldots, y_{l}$ as functions of $y_{1}, y_{l+1}, \ldots, y_{n}$ for $\left|y_{1}\right|<\rho,\left|y_{l+1}\right|<\rho, \ldots,\left|y_{n}\right|<$ $\rho$ where $\rho$ is a sufficiently small real number:

$$
y_{k}=y_{k}\left(y_{1}, y_{l+1}, \ldots, y_{n}\right), \quad k=2,3, \ldots, l .
$$

Therefore, for arbitrary $y_{1}(0), y_{l+1}(0), \ldots, y_{n}(0)$ such that $\left|y_{1}(0)\right|<\rho,\left|y_{l+1}(0)\right|<\rho, \ldots \ldots,\left|y_{n}(0)\right|<\rho$, we can find $y_{2}(0), \ldots, y_{l}(0)$ for which the corresponding solution has the desired property $y_{1}^{\prime}(0)=y_{1}^{\prime \prime}(0)=\ldots=$ $y_{1}^{(l)}(0)=0$.

Return to the linear system $y^{\prime}=A y$ considered in Example 1. This system consists of three equations with constant coefficients. Its general solution is

$$
y_{1}=c_{1} e^{x}+c_{2} e^{-x}, \quad y_{2}=c_{3} e^{x}, \quad y_{3}=c_{1} e^{x}-c_{2} e^{-x} .
$$

It has already been mentioned that the 2-rank $l$ of this system is 3 and the only admissible partition of $l$ is $(2,1)$. According to Proposition 4 , for any $a_{1}$ and $a_{2}$, this system has a solution

$$
y_{1}=\frac{a_{1}}{2} e^{x}+\frac{a_{1}}{2} e^{-x}, \quad y_{2}=a_{2} e^{x}, \quad y_{3}=\frac{a_{1}}{2} e^{x}-\frac{a_{1}}{2} e^{-x}
$$

such that $y_{1}(0)=a_{1}, y_{1}^{\prime}(0)=0$, and $y_{2}(0)=a_{2}$.

Consider the partition $(1,2)$, which is inadmissible. If $a_{2} \neq 0$, then $y_{2}(0)=a_{2}$, which implies $y_{2}^{\prime}(0) \neq 0$ (because $y_{2}^{\prime}(x)=y_{2}(x)$ ).

This example shows the importance of the feasibility of the partition of $l$ in the condition of Proposition 4.

Remark 1. The solution $\left(y_{1}, \ldots, y_{n}\right)^{\mathrm{T}}$, which exists according to Proposition 4 , can be chosen such that it satisfies the conditions

$$
\left|y_{k}(0)\right|<M \rho, \quad k=m+1, m+2, \ldots, n,
$$

for a certain $M$, which depends on the initial system and is independent of $a_{1}, \ldots, a_{m}$. Again, we give a proof for $m=1$. Any system having the 1-rank $l$ can be transformed (using a linear transformation of the unknowns with a constant nonsingular $n$-by- $n$ matrix $T$ ) to a system in which the first $l-1$ equations coincide with (14) and $y_{1}$ does not change under the action of $T$. The absolute values of the other variables increase by a factor not exceeding $M$, where $M$ is the maximum of the absolute values of the entries of the matrix $T$. Therefore, the corresponding solution has property (16).

Remark 2. In the linear (not necessarily autonomous) case, Proposition 4 is valid in a more general form: there exists a solution such that $y_{i}^{(j)}(0)\left(i=1,2, \ldots, m, j=0,1, \ldots, l_{i}-1\right)$ have arbitrarily specified values. 


\section{DIFFERENCE SYSTEMS}

The results obtained in Sections 1-4 and 6 are also valid for difference systems if, in the functional case, the differentiation is replaced by the unit shift $E$ with respect to the independent variable: $E \varphi(x)=\varphi(x+1)$. In the abstract algebraic situation, $E$ can be any automorphism of the field $K$. The sequential shifts $f^{[0]}, f^{[1]}$, $\ldots$ of the linear form $f$ along the solutions of the system $E y=A y$ are defined similarly to the sequential derivatives in the differential case; by analogy with formulas (2), we have

$$
f^{[0]}=f, \quad f^{[i]}=A^{\mathrm{T}}\left(E f^{[i-1]}\right), \quad i=1,2, \ldots,
$$

where $E f^{[i-1]}$ is the result of the componentwise shift of the column vector $f^{[i-1]}$ (again, we identify every linear form with the column vector of its coefficients in the basis $\left.y_{1}, \ldots, y_{n}\right)$.

Example 5. Consider the system $E y=A y$ with

$$
A=\left(\begin{array}{cccc}
1 & -\frac{1}{x(x+1)^{2}} & -x & 1 \\
1 & \frac{x^{2}+2 x-1}{x(x+1)} & x & -1 \\
1 & 0 & 1 & 0 \\
x+1 & 0 & 0 & \frac{x+1}{x}
\end{array}\right),
$$

$y=\left(y_{1}, y_{2}, y_{3}, y_{4}\right)^{\mathrm{T}}$, and $K=\mathbb{Q}(x)$. Assume that we are interested in the rational $y_{1}$ and $y_{2}$. The general rational solution of the initial system is the product of an arbitrary constant and the vector $(0,0,1, x)^{\mathrm{T}}$. The 2-rank of this system is 3 , and we can construct the new system $E z=B z$, where

$$
B=\left(\begin{array}{ccc}
0 & 1 & 0 \\
-\frac{x^{4}+6 x^{3}+13 x^{2}+14 x+4}{x(x+1)(x+2)^{2}} \frac{2 x^{4}+11 x^{3}+21 x^{2}+17 x+4}{x(x+1)(x+2)^{2}} & \frac{3 x^{2}+6 x+2}{x^{2}(x+1)^{3}(x+2)} \\
2 & -1 & \frac{x^{3}+3 x^{2}+x-2}{x(x+1)^{2}}
\end{array}\right),
$$

$z=\left(z_{1}, z_{2}, z_{3}\right)^{\mathrm{T}}, z_{1}=y_{1}, z_{2}=E y_{1}, z_{3}=y_{2}$. The general rational solution of the latter system is the product of an arbitrary constant and the vector $(1 / x, 1 /(x+1), x+1)^{\mathrm{T}}$. Both components $y_{1}$ and $y_{2}$ of the solution to the initial system are rational functions if and only if $y_{1}=c / x$ and $y_{2}=c(x+1)$, where $c$ is a constant.

In the difference case, as well as in the differential one, the algorithms for solving the systems are divided into two types - those based on reducing the system to a scalar equation of order $n$ and direct algorithms. For the case when $K$ is the field of rational functions, among the direct algorithms are the ones designed for the search for rational solutions [14, 10] and for the search for formal regular and irregular solutions [15]. As in the differential case, the algorithms for finding Liouville's solutions (see [16]) are designed for the scalar case; for the multidimensional case, uncoupling is used. The algorithms for finding solutions in the form of hypergeometric terms and their linear combinations $[17,18]$ are also designed for the scalar case. However, as claimed by its author, the idea behind the algorithm described in [18] can also be used in the multidimensional case.

In the difference case, Proposition 4 states that, if the $m$-rank of a difference autonomous system is $l$ and $\left(l_{1}, \ldots, l_{m}\right)$ is an admissible partition of $l$, then there exists a $\rho(0<\rho<r)$ such that, for any $a_{1}, \ldots, a_{m}$ satisfying the inequalities $\left|a_{i}\right|<\rho(i=1,2, \ldots, m)$, the system has a solution $\left(y_{1}, \ldots, y_{n}\right)^{\mathrm{T}}$ for which

$$
y_{i}(0)=a_{i}, \quad i=1,2, \ldots, m,
$$

and

$$
E^{j} y_{i}(0)=0, \quad i=1,2, \ldots, m, \quad j=1,2, \ldots, l_{i}-1
$$


that is,

$$
y_{i}(0)=a_{i}, \quad y_{i}(1)=\ldots=y\left(l_{i}-1\right)=0, \quad i=1,2, \ldots, m .
$$

It is also possible to use $\Delta=E-1$ instead of $E$, which leads to another solution satisfying the conditions

$$
\Delta^{j} y_{i}(0)=0, \quad i=1,2, \ldots, m, \quad j=1,2, \ldots, l_{i}-1
$$

that is,

$$
y_{i}(0)=y_{i}(1)=\ldots=y_{i}\left(l_{i}-1\right)=a_{i}, \quad i=1,2, \ldots, m .
$$

\section{ADJUSTABLE ALGORITHM}

The algorithms for constructing the new system with the matrix $B$ of the order coinciding with the $m$ rank of the initial system in the differential and the difference cases have much in common. They can be described in a unified way using the Ore noncommutative polynomials (the use of such polynomials in computer algebra is described, for example, in $[19,20])$. For this reason, the algorithm can also be used in the $q$-difference case. In such a generalized form, the algorithm was implemented by D.E. Khmel'nov in Maple. The matrices $B$ for the systems considered in Examples 2 and 5 can be constructed by invoking the same procedure, which is adjusted to one or another ring of Ore polynomials using some special parameters (in the case of Examples 2 and 5, the procedure is adjusted to the differential and the difference cases, respectively).

\section{ACKNOWLEDGMENTS}

This work was supported by the Eco-Net Program of the Ministry for Foreign Affairs of the French Republic (project no. 08119TG) and by the Russian Foundation for Basic Research (project no. 04-0100757).

We are grateful to S.Ya. Stepanov (Dorodnicyn Computing Center, Russian Academy of Sciences) and to M. Barkatou (University of Limoges) for consultations concerning the partial stability of solutions and differential consequences of systems. We are also grateful to M. van Hoeij (University of Florida) for the explanations concerning the direct application of the algorithm described in [18] to systems of difference equations.

\section{REFERENCES}

1. M. Bronstein, "Computer Algebra Algorithms for Linear Ordinary Differential and Difference Equations," in Progress in Mathematics (Birkhauser, Basel, 2001), Vol. 202, pp. 105-119.

2. M. F. Singer and M. van der Put, Galois Theory of Linear Differential Equations, Ser. Grundlehren der mathematischen Wissenschaften (Springer, Heidelberg, 2003), Vol. 328.

3. E. A. Coddington and N. Levinson, Theory of Ordinary Differential Equations (McGraw-Hill, New York, 1955; Inostrannaya Literatura, Moscow, 1958).

4. M. A. Barkatou and E. Pfluegel, "An Algorithm Computing the Regular Formal Solutions of a System Linear of Differential Equations," J. Symbolic Comput. 28, 569-587 (1999).

5. S. A. Abramov, M. Bronstein, and D. E. Khmelnov, "On Regular and Logarithmic Solutions of Ordinary Linear Differential Systems," Proceedings of the International Conference on Computer Algebra in Scientific Computing (CASC'05) Lect. Notes in Comput. Science (Springer, Heildelberg, 2005), Vol. 3718, pp. 1-12.

6. V. I. Vorotnikov and V. V. Rumyantsev, Stability and Control with Respect to a Part of Coordinates of the Phase Vector of Dynamical Systems: Theory, Methods, and Applications (Nauchnyi Mir, Moscow, 2001) [in Russian].

7. V. I. Vorotnikov and V. P. Prokop'ev, "On the Stability of Motion with Respect to a Part of Variables for Linear Systems," Prikl. Mat. Mekh. 42, 268-271 (1978).

8. A. G. Chermenskii, "Observability and Stability with Respect to a Part of Variables," Differ. Uravn. 23, 680-685 (1987).

9. W. R. Wasow, Asymptotic Expansions for Ordinary Differential Equations (Dover, New York, 1965; Mir, Moscow, 1968).

10. S. A. Abramov and M. Bronstein, "On Solutions of Linear Functional Systems," in Proceedings of the International Symposium on Symbolic and Algebraic Computation (ISSAC'2001) (ACM, New York, 2001), pp. 1-6.

11. M. A. Barkatou, "On Rational Solutions of Systems of Linear Differential Equations," J. Symbolic Comput. 28, 547-567 (1999). 
12. E. Pfluegel, "An Algorithm for Computing Exponential Solutions of First-Order Linear Differential Systems," in Proc. ISSAC'1997 (ACM, New York, 1997), pp. 164-171.

13. F. T. Cope, "Formal Solutions of Irregular Differential Equations," Am. J. Math. 58, 130-149 (1936).

14. S. A. Abramov and M. A. Barkatou, "Rational Solutions of First-Order Difference Systems," in Proceedings of the International Symposium on Symbolic and Algebraic Computation (ISSAC'1998) (ACM, New York, 1998), pp. 124-131.

15. M. A. Barkatou and G. Chen, "Computing the Exponential Part of a Formal Fundamental Matrix of a Linear Difference System,” J. Difference Equat. Appl. 5, 117-142 (1999).

16. M. F. Singer and P. A. Hendriks, "Solving Difference Equations in Finite Terms," J. Symbolic Comput. 27, 239259 (1999).

17. M. Petkovšek, "Hypergeometric Solutions of Linear Recurrences with Polynomial Coefficients," J. Symbolic Comput. 14, 243-264 (1992).

18. M. Van Hoeij, "Finite Singularities and Hypergeometric Solutions of Linear Recurrence Equations," J. Pure Appl. Algebra 139, 109-131 (1999).

19. M. Bronstein and M. Petkovšek, "On Ore Rings, Linear Operators and Factorization," Programmirovanie, No. 1, 27-44 (1994).

20. M. Bronstein and M. Petkovšek, "An Introduction to Pseudo-Linear Algebra," Theor. Comput. Sci. 157, 3-33 (1996). 